\title{
Mechanisms for the metabolic success of bariatric surgery
}

\author{
Darleen A. Sandoval (D)
}

Department of Surgery, University of Michigan, Ann Arbor, Michigan

\section{Correspondence}

Darleen A. Sandoval, Department of Surgery, University of Michigan, Ann Arbor, MI.

Email: darleens@umich.edu

Funding information

National Institute of Diabetes and Digestive and Kidney Diseases, Grant/Award Number: DK107282; Medlmmune; Novo Nordisk; Zafgen
To date, bariatric surgery remains the most effective strategy for the treatment of obesity and its comorbidities. However, given the enormity of the obesity epidemic, and sometimes variable results, it is not a feasible strategy for the treatment of all obese patients. A simple PubMed search for 'bariatric surgery' reveals over 28000 papers that have been published since the 1940s when the first bariatric surgeries were performed. However, there is still an incomplete understanding of the mechanisms for the weight loss and metabolic success of surgery. An understanding of the mechanisms is important because it may lead to greater understanding of the pathophysiology of obesity and thus surgery-alternative strategies for the treatment of all obese patients. In this review, the potential mechanisms that underlie the success of surgery are discussed, with a focus on the potential endocrine, neural and other circulatory factors (eg, bile acids) that have been proposed to play a role.

\section{KEYWORDS}

bariatric surgery, bile acids, metabolism

\section{1 | INTRODUCTION}

Bariatric surgery is currently our most effective strategy for sustained weight loss and improvements in the metabolic co-morbidities of obesity. Weight loss averages $\geq 30 \%$ over 10 years and leads to a $40 \%$ remission of type 2 diabetes mellitus (T2DM), ${ }^{1,2}$ often allowing complete discontinuation of T2DM-directed medications. No other treatment can claim this kind of remission of T2DM. The most popular bariatric surgeries worldwide are vertical sleeve gastrectomy (VSG) and Roux-en-Y gastric bypass (RYGB). ${ }^{3}$ After RYGB, ingested food bypasses approximately $95 \%$ of the stomach, the entire duodenum and a short portion of the jejunum. VSG, a distinct surgery in which $80 \%$ of the stomach along the greater curvature is removed with no intestinal rearrangement, is the most common bariatric procedure performed in the USA (58 vs $19 \%$ of total surgeries for VSG vs RYGB, respectively ${ }^{4,5}$ ) (for a schematic of each surgery, see Figure 1). Regardless of the success of bariatric surgery, the invasiveness and the infrastructure required to perform surgery continues to drive the need to find alternative strategies for treating obesity and T2DM.

The most simplistic hypothesis for the benefits of VSG is that a smaller stomach physically restricts meal size, leading to weight loss and improved metabolic endpoints secondary to weight loss. However, data in humans and rodents both demonstrate changes in feeding behaviour that go beyond mechanical restriction of meal size. Indeed, we have generated considerable data in our rodent model of VSG that challenge this hypothesis. We find sustained weight loss and decreased body fat but preserved lean mass with VSG alongside an early post-operative reduction in food intake. ${ }^{6}$ Although the reduction in food intake might suggest a restrictive mechanism, food intake returns to the level of sham animals approximately 2 weeks post-operatively. Furthermore, if exposed to a period of food-restricted weight loss, VSG animals become as hyperphagic as sham animals when returned to ad lib access to food. ${ }^{6}$ In another physiological model of hyperphagia, lactating female rodents who have had VSG increase feeding to the same extent as sham surgery females. ${ }^{7}$ These latter two studies demonstrate that any hypothesised physical restriction does not prevent hyperphagia when the physiology demands it. Lastly, VSG animals consistently avoid calorically-dense high-fat foods, indicating that they are not attempting to overcome physical restriction by choosing more calorically-dense foods. ${ }^{8,9}$ In humans ${ }^{10,11}$ and rodents, ${ }^{12}$ nutrients rapidly empty from the pouch or sleeve from RYGB and VSG surgeries, respectively, further suggesting that mechanical restriction does not 


FIG URE $\mathbf{1}$ The impact of vertical
sleeve gastrectomy (VSG) and Roux-en-Y
gastric bypass (RYGB) on the central
nervous system (CNS), gut and gut-brain
axis. The CNS is more highly activated by
surgery and induces changes in feeding
patterns and macronutrient preference.
The gut responds with increases
postprandial peptide secretions, increases
in plasma bile acids (BA) and changes in
the microbiome. The gut-brain axis may
integrate these two systems as celiac
gangliectomy blunts weight loss responses
to RYGB

play a role in surgery-induced reductions in feeding. Thus, the data suggest that the mechanisms that drive the success of bariatric surgery are physiological in nature. This review will discuss the potential mechanisms that underlie the success of surgery, with a focus on what has been learned from rodent models.

\section{2 | COMPARISONS OF CLINICAL AND PRECLINICAL OUTCOMES}

Preclinical work offers the ability to study cellular and molecular mechanisms that drive the success of surgery. However, there are always important issues to consider when comparing rodent with clinical data. Despite this, the outcomes of surgery are qualitatively very similar between rodents and humans.

Physiologically, both humans and rodents have sustained reductions in body mass, changes in feeding behaviour including meal patterning, food reward and macronutrient preference, rapid nutrient entry into the intestine, large postprandial increases in gut peptides including glucagon like peptide-1 (GLP-1), increases in circulating bile acids (BA) and changes in the microbiome (see below for details). That being said, there are potential critical differences. Most simplistically and not surprisingly, the timing of changes in body weight and food intake differs. The body mass and food intake nadir is typically 2-3 weeks in rodents and 6-12 months in humans. The majority of this weight loss in rodents is fat, rather than lean mass, ${ }^{6}$ whereas humans lose both lean and fat mass. ${ }^{13}$ With regard to food intake, humans appear to have sustained reductions in food intake that are reported up to 10 years post-operatively, ${ }^{14}$ whereas rodents return to ingesting similar caloric loads as sham surgery animals 2-3 weeks after surgery. ${ }^{6}$ However, the persistently lower food intake in humans may actually reflect the intake that is appropriate to maintain energy balance for the new lower body mass. It is also worth noting that the assessment of food intake in humans is confounded not only by experimental error given that dietary recall consistently results in under-reporting, but also by the fact that patients undergo preand post-surgical feeding behaviour counselling. Thus, in humans, the ability to understand the biological impact of surgery on food intake is limited.

Regardless, if we assume that food intake is persistently reduced in humans, then another species difference is highlighted by recent work in mice suggesting that the maintenance of weight loss in mice after RYGB may be largely a result of increased energy expenditure rather than reduced food intake. ${ }^{15}$ By contrast, changes (either increases or decreases) in energy expenditure are not consistently reported after VSG in rodents. ${ }^{6,16}$ This, again, is in contrast to humans who have reported reductions in energy expenditure after both RYGB ${ }^{17}$ and VSG. ${ }^{18}$ However, this reduction in energy expenditure could be in response to the reduced caloric intake or it may reflect surgery-induced changes in body composition rather than a direct effect of surgery on energy expenditure. ${ }^{13}$ That being said, assessment and interpretation of changes in energy expenditure are constantly being debated in both humans and rodents. Regardless of the challenges, there is value in using animal models when aiming to understand the biological impact of surgery on these endpoints and when generating targets for the mechanisms underlying the success of surgery.

\section{3 | MECHANISMS FOR METABOLIC SUCCESS}

\subsection{The role of gut-secreted peptides}

That a change in gastrointestinal (GI) anatomy could cause such a rapid and sustained weight loss with the associated improvements in co-morbidities highlights the tremendous impact of the GI system with respect to regulating homeostasis. However, the exact mechanism(s) driving the weight loss and metabolic improvements 
still remain elusive. One hypothesis that persists is that bariatric surgery increases the nutrient-induced secretions of Gl-tract peptides shown to play a role in the regulation of appetite, energy expenditure and blood glucose homeostasis.

\section{2 | Satiety-regulating peptides}

Specialised enteroendocrine cells secrete peptides in response to changes in nutrient status. Glucose-dependent insulinotropic peptide (GIP) and GLP-1 secreted from predominantly the upper and lower small intestine, respectively, are considered to be important for the regulation of glucose homeostasis, whereas cholecystokinin (CCK), GLP-1, glucagon like peptide-2 (GLP-2), oxyntomodulin and peptide $Y Y$ (PYY) also function as satiety signals. ${ }^{20} \mathrm{GIP}$ is secreted from enteroendocrine K-cells located within the proximal gut and is critical for regulation of insulin and gastric secretion and motility. Although, traditionally, these enteroendocrine cells were considered to be differentiated by the peptides they secrete, it is most likely that the differentiation is regional. ${ }^{21-23}$ An example of regional distinction is that GLP-1-secreting cells in the distal jejunum and ileum coexpress PYY, ${ }^{24}$ whereas proximal GLP-1-secreting cells co-express CCK, GIP, neurotensin, or secretin. ${ }^{25}$ Although, traditionally, these enteroendocrine cells were differentiated by the peptides they secrete, recent research suggests that there is heterogeneous co-expression of different peptides in these cells, and that these patterns of co-expression differ between regions of the gGI tract. ${ }^{26}$ This process may enable enteroendocrine cells to respond to specific local nutritional stimuli. ${ }^{27}$ Bariatric surgeries change not only the $\mathrm{Gl}$ anatomy, but also the rate at which nutrients enter the intestine. Thus, it is not surprising that many of these gut peptides are also altered by bariatric surgery.

Ghrelin is secreted from gastric and duodenal enteroendocrine cells; it is one of the few GI tract-secreted peptides for which circulating levels decrease postprandially. Increased circulating ghrelin levels are associated with an increased, rather then a reduced, drive to eat. ${ }^{28}$ With RYGB, ghrelin levels are maintained in many studies, whereas, with VSG, they are consistently decreased, ${ }^{29,30}$ suggesting that, although the stomach and duodenum are no longer receiving luminal nutrient stimuli with RYGB, the blood flow to the tissue is sufficient to stimulate the release and maintain plasma ghrelin levels. To determine whether the decrease in ghrelin with VSG is necessary for the success of surgery, VSG was performed in mice genetically devoid of ghrelin. ${ }^{31}$ However, these mice lost body weight and improved their glucose tolerance as much as sham surgery controls. Together with the slightly superior improvements in body weight and glucose homeostasis with RYGB vs VSG, these data suggest that a reduction of ghrelin, in and of itself, is not necessary for metabolic improvements after bariatric surgery.

It is not clear whether CCK, an anorectic peptide secreted from the upper $\mathrm{GI}$ tract, increases after bariatric surgery. Although CCK has been found to be increased in RYGB, this increase appears to be greater after VSG in humans. ${ }^{32,33}$ However, Otsuka Long-Evans Tokushima Fatty rats, lacking CCK-1 receptors, are able to lose weight and improve glucose homeostasis in response to RYGB, ${ }^{34}$ suggesting that CCK signalling is also not necessary for the metabolic success of bariatric surgery, or at least in response to RYGB.

Peptide YY and GLP-1 are secreted from distal L-cells and both peptides increase postprandially after VSG and RYGB in humans and rodents. ${ }^{10,35-39}$ Although consistent increases in PYY are seen after surgery, its mechanistic role in the weight loss associated with bariatric surgery has not been studied as extensively as that of GLP-1. One study has shown that PYY knockout (KO) mice lost less weight acutely after RYGB (assessment at 10 days post-operatively). ${ }^{40}$ Unfortunately, these mice were not assessed further for changes in feeding behaviour.

Both total and active levels of GLP-1 are increased after surgery. Postprandial GLP-1 levels are strikingly (approximately 10-fold) increased after both RYGB and VSG and this increase is seen within 2 days, and is maintained for at least 2 years after surgery. ${ }^{10,33,41-43}$ Importantly, weight loss through caloric restriction does not lead to an increase in postprandial GLP-1 levels such as for VSG and RYGB, ${ }^{43}$ highlighting the physiological effect of these surgeries. Preproglucagon is the gene that produces GLP-1, although it also produces other peptides and based on posttranslational processing this occurs in a tissue-specific fashion. In the intestine and central nervous systerm (CNS), expression of prohormone convertase $1 / 3$ processes preproglucagon peptides to produce GLP-1 and oxyntomodulin, which are both assumed to regulate satiety and glucose homeostasis, and GLP-2, which regulates intestinal growth and morphology. Circulating levels of all of these peptides are increased by bariatric surgery. ${ }^{36,44,45}$ Preclinical studies in rats demonstrate that the increase in GLP-2 occurs in parallel with intestinal hypertrophy after RYGB. ${ }^{46}$ However, mice null for the GLP-2 receptor lose weight and improve glucose tolerance similar to wild-type animals in response to VSG. ${ }^{47}$ Given its link to glucose sensing and absorption in the gut, ${ }^{48}$ the increase in GLP-2 with RYGB could also blunt some of the macronutrient malabsorption that would be expected to increase with intestinal rearrangement, an effect minimised with VSG. Thus, these apparent differences could reflect the varied impact of the specific surgeries or it could simply be that the increase in GLP-2 and consequent increase in hypertrophy that occurs with RYGB is a marker but not a mechanism of the success of surgery.

Peptide $Y Y$ is activated by a cleavage enzyme, dipeptidyl peptidase-4 (DPP4). DPP4 also degrades and inactivates GLP-1. To determine the role of these two anorectic peptides in the regulation of feeding after RYGB, one study administered, saline, a DPP4 inhibitor, a GLP-1 receptor antagonist, or a combination of the DPP4 inhibitor plus the GLP-1 receptor antagonist to patients 3 months after RYGB. ${ }^{49}$ Only the combined drugs significantly increased the amount of food ingested during the standardised meal, suggesting that both PYY and GLP-1 receptor signalling are necessary to regulate acute meal ingestion after surgery. Altogether, these data suggest that the combined impact of these anorectic peptides is more important than the impact of any one peptide alone. 
In the pancreas, predominant expression of proconvertase 2 leads to preproglucagon processing to produce glucagon. Although some studies have reported an increase in postprandial glucagon after RYGB, ${ }^{50-53}$ a later study suggested that this work was confounded by the fact that RYGB causes large increases glicentin, another preproglucagon peptide that has increasing cross-reactivity with standard glucagon enzyme-linked immunosorbent assays with increasing plasma concentrations. ${ }^{54}$ Thus, more research is needed from independent groups utilising sensitive and specific assays to determine whether glucagon is increased with surgery or not. Regardless, if glucagon does increase, it may not be critical in the success of surgery because genetic deficiency of both the glucagon receptor and the GLP-2 receptor does not blunt the metabolic benefits of VSG. ${ }^{47}$

Oral glucose drives a much greater insulin response compared to when the same glucose load is administered i.v. ${ }^{55}$ This incretin effect is attributed to GLP-1 and GIP. ${ }^{56,57}$ In healthy and T2DM subjects, GLP-1 and GIP contribute almost equally to the incretin effect stimulating the majority of postprandial insulin release. ${ }^{56}$ Instead, the defect with obesity and T2DM appears to be in an overall reduction in the incretin effect. ${ }^{58}$ Both RYGB and VSG correct and even enhance the incretin effect. Both GLP-1 and GIP are also rapidly degraded by DPP4, the same peptide that activates PYY. DPP4 inhibitors increase GIP and GLP-1 two-fold. When administered with or without exendin 9-39, DPP4 inhibitors improve glucose tolerance and insulin secretion in non-surgical T2DM patients. ${ }^{59}$ In mice, DPP4 inhibitors retain their glucose improvement efficacy when either GLP-1R or GIPR are genetically deficient but not in double GLP-1R/GIPR KO mice. $^{60}$ Together, these data suggest that either GLP-1 or GIP receptor signalling is sufficient for the ability of DPP4 inhibitors to improve glucose tolerance. However, in T2DM patients that have had RYGB a DPP4 inhibitor failed to improve glucose tolerance or $\beta$-cell function when GLP-1 receptor signalling was blocked, ${ }^{61}$ suggesting that RYGB shifts the balance of the incretin effect toward GLP-1 and away from GIP. Interestingly, GIP does not show consistent increases after RYGB ${ }^{43,62}$ and even has demonstrated decreases 1 year after both RYGB ${ }^{63}$ and VSG. ${ }^{30}$ Together, these data suggest that postprandial increases in GLP-1 are more important than GIP with respect to regulating the changes in postprandial insulin and consequently glucose after bariatric surgery.

Despite the indication that GLP-1 is important for postprandial changes in glucose homeostasis, whether the increase in GLP-1 is necessary for weight loss or T2DM resolution remains to be determined. One of the complications of determining the mechanistic role of GLP-1 in mediating T2DM resolution is that the duration of disease and, consequently, the degree of impairment of $\beta$-cell function prior to surgery may be more critical in determining whether those $\beta$-cells can recover sufficiently to resolve T2DM. ${ }^{64}$ Although one study found a predictive role of the degree of increase in GLP-1 and in the remission of T2DM after RYGB, ${ }^{39}$ another study found no such relationship after VSG. ${ }^{65}$ Still, administration of the GLP-1 receptor antagonist, exendin 9-39, impaired the insulin response to an oral glucose load in both humans and rodents after bariatric surgery, ${ }^{41,66-68}$ suggesting a role for GLP-1 in postprandial insulin secretion. However, in dietary-induced obese mouse models genetically deficient in GLP-1 receptors, both VSG and RYGB retain their ability to induce weight loss and improve glucose. ${ }^{69-71}$ Lastly, inducible knockdown of the $\beta$-cell GLP-1 receptor in adult mice using the Cre-loxP system prevented improvements in glucose tolerance and glucose-stimulated insulin secretion but not weight loss ${ }^{72}$ in one study, although there was no impact of a similar genetic disruption on VSG results in another. ${ }^{73}$ A recent study with data from lean postgastrectomy patients with postprandial hypoglycaemia and a lean VSG mouse model confirms previous studies that pharmacological blockade of GLP-1 receptor signalling increases glucose and reduces postprandial insulin responses. ${ }^{74}$ Although it is true that impaired insulin resistance could confound the ability to detect a role of GLP-1 in surgical success in mice, there are several problems with extrapolating these recent data to suggest that GLP-1 is critical for T2DM resolution. First, the extent to which the altered glucose responses to a meal after surgery are responsible for T2DM is not clear. Indeed, it has been argued that an increase in glucose variability as is seen with bariatric surgery has detrimental effects, including an increased cardiovascular risk. ${ }^{75}$ The other issue is the interpretation of pharmacological data. Blockade of GLP-1 receptor signalling increases the glucose curve in both sham and surgery animals or control vs RYGB patients. In one clinical study where RYGB patients were treated with Ex9 during a meal, the glucose area under the curve values of the Ex9-treated patients were expressed relative to vehicle and the impairment was not statistically different between control and RYGB patients. ${ }^{76}$ Thus, the interpretation of these pharmacological studies is complicated and leaves open the question of whether GLP-1R signalling matters specifically for T2DM resolution after surgery, or whether it just generally matters for insulin regulation whether the patients have had surgery or not.

Although it is surprising that the 10 -fold increase in plasma GLP-1 with surgery might not play a critical mechanistic role in the success of surgery, it is possible that this increase reflects a defensive response of the intestine to the rapid nutrient entry induced by both surgeries. Clearly, the changes in GI anatomy with surgery greatly alter the cocktail of postprandial gut peptides. Although these changes, in particular with GLP-1, have been found to be associated with greater weight loss, association does not mean causation. Alternatively, it may be that changes in the whole cocktail of gut peptides are necessary for the response to bariatric surgery, explaining why genetic removal of signalling for one gut peptide at a time has a minimal effect. More work will be needed to understand whether the changes in these gut peptides are a marker or a mechanism for the success of surgery.

\subsection{The role of the nervous system}

Feeding behaviour is carefully regulated by the CNS and, given the clear changes in feeding behaviour with surgery, it would follow that the CNS is mediating these changes. Feeding patterns are clearly and persistently altered by bariatric surgery with both humans and 
rodents ingesting smaller more frequent meals post-operatively. ${ }^{77-83}$ In addition, bariatric surgery alters taste sensitivity, food reward and macronutrient preference in rodents. ${ }^{8,9,84,85}$ With regard to the latter, lean and obese rats and mice will overwhelmingly ingest fat when given a choice between fat, carbohydrate and protein macronutrients. ${ }^{6,9}$ However, bariatric surgery shifts this preference towards carbohydrate and away from fat. ${ }^{6,9,85,86}$ In humans, similar shifts in food preference are observed. ${ }^{87}$ An interesting possibility is that the reduced appetite or shift in macronutrient preference seen with bariatric surgery is not because some foods are found to be more favorable than others but, instead, because ingestion of certain foods leads to aversive side-effects. Many patients report feelings of food-induced sickness after either RYGB or VSG. ${ }^{88,89}$ Indeed, greater weight loss is correlated with reports of greater food-induced aversion. ${ }^{89}$ Similarly, rats have a particular aversion to oil after both RYGB ${ }^{90}$ and VSG.

In addition to changes in feeding behaviour, there are multiple points of data that indicate that the brain is more highly activated after a meal following bariatric surgery. For example, we have found that FOS-like immunoreactivity, a marker for neuronal activation, within a specific area of the hindbrain, the nucleus of the solitary tract (NTS) and the area postrema, increases after a sucrose or an equi-caloric lipid gavage to a greater extent in male rats that have had VSG vs rats that had sham surgery and were either ad lib or pair-fed to the VSG animals. ${ }^{8}$ The NTS and area postrema, are critical junctures between the vagus and blood stream, respectively. Indeed, data suggest that it is not just the signalling to this region that is altered but that the electrical properties of neurones within the NTS that are altered by high fat diet and this effect is reversed by RYGB. ${ }^{91}$ In patients that have received RYGB, the hypothalamus, pituitary and medial orbital cortex were all more highly activated and the right dorsolateral frontal cortex were more deactivated after a meal. $^{92}$

A critical question is what are the key signals that drive this increase in CNS activation with surgery? The increase in circulating hormones/gut peptides (many of which have receptors throughout the CNS) and/or nutrients could be acting directly within the CNS to initiate these responses. However, currently, there are limited data to suggest that direct hormone and/or nutrient action drives greater CNS activation. Although GLP-1 receptor expression within the CNS has been shown to be important for regulation of body mass, CNS administration of exendin 9-39, a potent GLP-1 receptor antagonist, in rats does not block the impact of RYGB on weight loss, ${ }^{71}$ suggesting that CNS GLP-1 receptor signalling is not critical for surgery-induced weight loss. However, it is possible that peripheral nerve GLP-1 receptor signalling overrides the CNS antagonism and/or that GLP-1 receptor signalling works in concert with other gut peptides (eg, PYY) to regulate feeding. In addition, because of its increased 'gastric' or sleeve emptying rate, nutrients enter the intestine much more rapidly. This clearly changes the pattern of nutrient responses to a meal. For example, continuous glucose monitoring in patients after RYGB shows a greater dynamic range in glucose levels with larger peaks but rapid returns to baseline after a carbohydrate-rich meal. ${ }^{93}$ Whether these greater peaks could contribute to greater postprandial CNS activation remains to be determined.

Another possibility is that the nutrient levels themselves or the concomitant rise in gut peptides increase vagal afferent firing, which then feeds back to higher brain centres. Additionally, the increased gastric pressure that drives the increase in emptying rate with $\mathrm{VSG}^{12}$ could provide greater mechanical feedback via the vagus to the CNS. A standard approach for examining the neuronal component of the gut-brain axis is to surgically ablate the vagus. Neither hepatic branch, ${ }^{94}$ nor subdiaphragmatic ${ }^{95}$ vagotomy impact surgical weight loss. However, subdiaphragmatic vagotomy did blunt surgery-induced shifts in taste preference, and the mechanism is considered to be a result of alterations of dopamine signalling within the CNS. ${ }^{95}$ In addition, when the vagus is ligated at the stomach, RYGB is less effective than when the nerve is left intact. ${ }^{96}$ Lastly, ablation of the vagal branch that innervates the intestine (celiac branch vagotomy) also blunts surgery-induced weight loss and suppression of feeding in response to RYGB. ${ }^{97}$ Altogether, these data support a specific role for intestinal rather than hepatic vagal innervation in the success of surgery. Similar to distinct nuclei within the CNS, the vagus is a heterogeneous population of neurones, ${ }^{98-100}$ allowing individual neurones to respond to distinct stimuli. Indeed, activation of specific neurones within the nodose ganglia, the cell body of the vagus, have been found to differentially regulate GI functions. For example, optogenetic activation of vagal neurones that express GLP-1 receptors regulate gastric stretch, whereas activation of neurones expressing a specific nutrient sensing G-coupled protein receptor, GPR65, regulate intestinal nutrient sensing. ${ }^{100}$ The application of this technology to surgery will be an important to move towards a better understanding of the role of the vagus in mediating the various physiological responses to bariatric surgery.

\subsection{The role of intestinal morphology}

The intestine forms a critical barrier from the external to internal environment. Perhaps because of this critical function, there is a very high turnover (every 4 days) of the epithelial cells that make up this barrier. These cells line the villi (absorptive region) and crypts (the region where the stem cells, the precursors for intestinal epithelial cells, are located). Nutritional state and intestinal diseases both impact intestinal morphology (villi length and/or crypt depth); for example, obesity has been found to increase both of these variables. ${ }^{101}$ However, RYGB has also been found to increase overall intestinal thickness, both villi length and width, crypt depth, and mucosa volume within the roux and common but not in the biliopancreatic limb. ${ }^{46,102-104}$ The lack of proliferation in the biliopancreatic limb suggests a role of nutrient exposure (or lack thereof) in directing these regional differences. Interestingly, data suggest that, after RYGB, the intestine directs glucose towards the hexosamine biosynthesis pathway, a metabolic pathway critical for tissue growth. ${ }^{105}$

The impact of VSG on intestinal morphology is less clear. One paper reported no impact of VSG on intestinal morphology ${ }^{102}$ and 
other studies demonstrate an increase in villus length but not crypt depth. ${ }^{106-108}$ Also, unlike RYGB, VSG increases the number of GLP-1 positive cells within the jejunum and ileum. ${ }^{106,108}$ This would suggest that VSG drives an increase in production of GLP-1 positive cells. Given that the plasma levels of other gut peptides (CCK, GIP) are also increased by VSG, these data suggest that there is an overall increase in enteroendocrine cell production. However, more work is needed to differentiate the impact of changes in the response of these enteroendocrine cells to nutrients vs the increase in cell number and their respective contribution to the overall increase in gut peptide levels and of course whether these differences matter to the overall success of surgery.

\subsection{The role of changes in BA}

Bile acids are synthesised by the liver and travel from the liver to the gall bladder, bile duct, intestine, blood and, finally, are transported back into the liver. Primary BA produced by the liver can be conjugated with either glycine or taurine. Once in the lumen of the intestine, the intestinal flora modifies primary BA to form secondary BA. Changes in BA have been linked to changes in glucose homeostasis. For example, fasting plasma levels and specifically increased levels of cholic acid, deoxycholic acid and their conjugated forms are found in insulin resistant patients ${ }^{109}$ and elevated fasting levels of total BA with preferential increases in more hydrophobic and conjugated BA are found in T2DM patients. ${ }^{109-111}$ Interestingly, RYGB patients who have profound improvements in glucose homeostasis also have been found to have a more than three-fold increase in plasma BA compared to weight-matched non-surgical controls. ${ }^{112}$ Specifically, RYGB in humans increases cholic acid (CA), chenodeoxycholic acid (CDCA) (primary BA) and deoxycholic acid (a secondary BA). ${ }^{112-116}$ The difference in $B A$ between bariatric surgery and impaired glucose metabolism may reside in differences in the ratio of the various BA species. For example, one study demonstrated that a higher proportion of CDCA relative to total BA (CDCA\%) and a shorter duration of diabetes was predictive of surgery-induced remission of T2DM in Chinese patients. ${ }^{117}$ Similar effects on BA increases are observed after bariatric surgery in animal models. ${ }^{107,118,119}$ In mice, VSG also results in a change in the composition of $\mathrm{BA}$ also towards $\mathrm{CA}$, although there is also an increase in tauroursodeoxycholic acid, ${ }^{93}$ a particular BA that has been found to have potent metabolic effects in a diabetic mouse model. ${ }^{120}$ Interestingly, ursodeoxycholic acid, a hydrophilic secondary BA utilised pharmacologically to treat cholestasis, has no additional impact on gut peptide or glucose levels when administered to RYGB patients. ${ }^{121}$ Thus, the differences in the impact of obesity and bariatric surgery are important because different types of BA have differing metabolic properties and differing affinities (including antagonistic properties) for the two receptors thought to be critical for BA signalling.

In addition to their emulsifying properties, BA also act as hormones with two different identified receptors; a cell surface membrane-bound G protein-coupled receptor (TGR5) ${ }^{122}$ and a nuclear transcription factor, farnesoid $X$ receptor (FXR). ${ }^{123,124}$ TGR5 activation within the intestine increases secretion of GLP-1 from intestinal L-cells ${ }^{125}$ and, within the muscle and brown adipose tissue, it increases energy expenditure. ${ }^{126} \mathrm{FXR}$, which is highly expressed in the liver, intestine, kidney and adrenal glands, has been found to be a crucial upstream regulator of lipid and glucose metabolism, as well as of BA synthesis. ${ }^{127-129}$

Intestinal activation of FXR results in the upregulation of fibroblast growth factor 19 (FGF19; FGF15 is the mouse orthologue) synthesis and secretion. In turn, circulating FGF19/15 regulates hepatic BA synthesis and stimulates gall bladder filling. FGF19/15 improves glucose homeostasis specifically by inhibiting hepatic gluconeogenesis, ${ }^{130}$ enhancing hepatic mitochondrial activity and glycogenolysis, ${ }^{131,132}$ and increasing insulin-independent glucose uptake, ${ }^{130}$ and does so by activating multiple fibroblast growth factor receptors in multiple target organs, including the liver, pancreas, adipose and brain. ${ }^{133}$ Demonstrating translational relevance of these findings, patients with T2DM have reduced FGF19 levels ${ }^{111}$ and RYGB increases FGF19, an effect that has been linked to the surgery-induced T2DM remission. ${ }^{114}$

Of course association does not mean causation and so preclinical studies have been carried out aiming to determine whether BA signalling is necessary for the success of surgery. With TGR5, the data are conflicting. One study found that TGR5-KO mice demonstrated similar weight loss compared to sham surgery animals but the degree of surgery-induced improvements in both glucose tolerance and hepatic triglycerides was blunted. ${ }^{135}$ These mice also retained the postprandial increase in GLP-1. Although another study reported that TGR5-KO mice had blunted improvements in glucose tolerance and hepatic triglycerides, it was also found that these mice did not lose weight and had blunted energy expenditure and postprandial increases in GLP-1. ${ }^{136}$ It is unknown what factors contribute to these differences. Both studies used mice that were generated by Merck, ${ }^{137}$ although mice were purchased from Taconic for one paper ${ }^{135}$ and were received directly from Merck ${ }^{136}$ in the other. There are also methodological considerations. The age of the animals when placed on the high-fat diet, the type and amount of time the animals were on high-fat diet, and the amount of time the animals were studied after surgery differed between the studies. This latter point might be important because both studies report early weight loss after VSG regardless of genotype but the TGR5KO animals that had VSG regained body weight in the paper by Ding et $\mathrm{al}^{136}$ at a time point later than the time point at which the animals were killed in the paper by McGavigan et al ${ }^{135}$ Lastly, although it is difficult to tell based on the reported methodology, differences in surgical technique could also contribute. Thus, clearly more work is needed to determine the role, if any, of TGR5 in the metabolic success of surgery.

The specific role of FXR in the metabolic success of surgery has also been explored. FXR-KO mice lost less weight and they did not improve glucose tolerance or shift their macronutrient preference to carbohydrates from fat in response to VSG ${ }^{118}$ Interestingly, the FXR KO mice preserved their postprandial GLP-1 response to surgery, suggesting that FXR does not regulate GLP-1 secretion and also that 
GLP-1 secretion alone cannot overcome the impact of loss of FXR on surgical outcome.

Downstream of FXR signalling within the liver is the small heterodimer partner pathway. Using a viral knockdown of this pathway, Myronovych et al ${ }^{107}$ found that, although VSG induced weight loss, there was a proinflammatory phenotype in these animals suggesting that VSG-induced improvements in hepatic lipid levels and inflammation is dependent upon small heterodimer partner. Given the wide impact and multi-target organ impact of FXR and FGF15/19 signalling on metabolism, more mechanistic preclinical work is needed to understand the full impact of this system on the success of bariatric surgery.

\subsection{The role of the microbiome}

A potential critical factor in integrating BA processing and FXR signalling is the microbiome. FXR indirectly regulates the microbiome by regulating BA synthesis. Conversely, as discussed above, the microbiome regulates the conversion of primary BA to secondary BA within the lumen of the intestine. However, recent work suggests that FXR also directly regulates the composition of the microbiome. ${ }^{138}$ In turn, the microbiome regulates hepatic cholesterol 7 alpha-hydroxylase (CYP7A1; the rate limiting enzyme in BA synthesis) and FGF15 in the ileum; an effect that is dependent upon FXR signalling. ${ }^{139}$ Clearly, these data highlight the very close symbiotic relationship between FXR signalling, the microbiome, and BA.

In both wild-type and FXR-KO mice, the expected shift in bacteroides genus to the firmicutes genus in the microbiome was seen after VSG suggesting that these flora change in an FXR-independent manner. ${ }^{118}$ However, the improvement in glucose homeostasis with VSG was associated with increases in a specific genus, roseburia, a butyrate producing bacterium, and this effect was not seen in FXR$\mathrm{KO}$ mice. Still, whether the changes in the microbiome with bariatric surgery drive the metabolic impact of surgery remains to be seen. A recent manuscript by Aron-Wisnewski et al $^{140}$ found that severe obesity is associated with low microbial gene richness. However, RYGB patients retained this low microbial gene richness despite weight loss and cardiometabolic improvements; even in a cohort that was studied 5 years after RYGB. These data dissociate microbial dysbiosis from metabolism and also emphasise that we are only at the beginning of our understanding of the impact of the microbiome on physiological regulation of body mass and certainly in the metabolic impact of bariatric surgery.

\section{4 | CONCLUSIONS}

A simple PubMed search for 'bariatric surgery' reveals over 28000 papers that have been published since the 1940s when the first bariatric surgeries were performed. Most of what has been learned from this extensive literature concerns bariatric surgery having a widespread physiological impact. This particular review has summarised some of the work that has explored the role of the CNS, the gut and the gut-brain axis in the responses to bariatric surgery (Figure 1). However, there is a still lack of understanding regarding the mechanisms that underlie the success of surgery. The most promising link in mice appears to be between BA and/or FXR signalling, although clearly more work is needed to understand the link between BA signalling and the metabolic success of surgery in humans.

\section{CONFLICT OF INTERESTS}

DAS has received research support from Novo Nordisk, Zafgen and Medlmmune. DAS has been a paid speaker for Novo Nordisk. Data sharing is not applicable to this article because no new data were created or analysed in this study.

ORCID

Darleen A. Sandoval (iD https://orcid.org/0000-0003-3669-3278

\section{REFERENCES}

1. Schauer PR, Kashyap SR, Wolski K, et al. Bariatric surgery versus intensive medical therapy in obese patients with diabetes. $N$ Engl J Med. 2012;366:1567-1576.

2. Schauer PR, Bhatt DL, Kirwan JP, et al. Bariatric surgery versus intensive medical therapy for diabetes -5 -year outcomes. $N$ Engl J Med. 2017;376:641-651.

3. Welbourn R, Hollyman M, Kinsman R, et al. Bariatric surgery worldwide: baseline demographic description and one-year outcomes from the fourth IFSO Global Registry Report 2018. Obes Surg. 2019;29:782-795.

4. American Society for Metabolic and Bariatric Surgery. Estimate of bariatric surgery numbers, 2011-2016 | American Society for Metabolic and Bariatric Surgery, https://asmbs.org/resources/estimate-of-bariatric-surgery-numbers. Accessed May 23, 2018.

5. Dimick JB, Nicholas LH, Ryan AM, et al. Bariatric surgery complications before vs after implementation of a national policy restricting coverage to centers of excellence. JAMA. 2013;309:792-799.

6. Stefater MA, Pérez-Tilve D, Chambers AP, et al. Sleeve gastrectomy induces loss of weight and fat mass in obese rats, but does not affect leptin sensitivity. Gastroenterology. 2010;138:2426-2436, 2436.e1-3.

7. Grayson BE, Schneider KM, Woods SC, et al. Improved rodent maternal metabolism but reduced intrauterine growth after vertical sleeve gastrectomy. Sci Transl Med. 2013;5:199ra112.

8. Chambers AP, Wilson-Perez HE, McGrath S, et al. The effect of vertical sleeve gastrectomy on food selection and satiation in rat. Am J Physiol Endocrinol Metab. 2012;303:E1076-E1084.

9. Wilson-Pérez HE, Chambers AP, Sandoval DA, et al. The effect of vertical sleeve gastrectomy on food choice in rats. Int J Obes. 2012;37:288-295.

10. Dirksen C, Damgaard M, Bojsen-Møller KN, et al. Fast pouch emptying, delayed small intestinal transit, and exaggerated gut hormone responses after Roux-en-Y gastric bypass. Neurogastroenterol Motil. 2013;25:346-e255.

11. Melissas J, Daskalakis M, Koukouraki S, et al. Sleeve gastrectomy - a 'food limiting' operation. Obes Surg. 2008;18:1251-1256.

12. Chambers AP, Smith EP, Begg DP, et al. Regulation of gastric emptying rate and its role in nutrient-induced GLP-1 secretion in rats after vertical sleeve gastrectomy. Am J Physiol Endocrinol Metab. 2014;306:E424-E432. 
13. Carey D, Pliego G, Raymond R. Body composition and metabolic changes following bariatric surgery: effects on fat mass, lean mass and basal metabolic rate: six months to one-year follow-up. Obes Surg. 2006;16:1602-1608.

14. Sjostrom L, Lindroos AK, Peltonen M, et al. Lifestyle, diabetes, and cardiovascular risk factors 10 years after bariatric surgery. $N$ Engl J Med. 2004;351:2683-2693.

15. Hao Z, Mumphrey MB, Townsend RL, et al. Body composition, food intake, and energy expenditure in a murine model of Rouxen-Y gastric bypass surgery. Obes Surg. 2016;26:2173-2182.

16. Feng Y, Zhong C, Niu J, et al. Effects of sleeve gastrectomy on lipid and energy metabolism in ZDF rats via PI3K/AKT pathway. Am J Transl Res. 2018;10:3713-3722.

17. Bosy-Westphal A, Braun W, Schautz B, et al. Issues in characterizing resting energy expenditure in obesity and after weight loss. Front Physiol. 2013;4:47.

18. Guida B, Cataldi M, Busetto L, et al. Predictors of fat-free mass loss 1 year after laparoscopic sleeve gastrectomy. J Endocrinol Invest. 2018:41:1307-1315.

19. Das SK, Roberts SB, McCrory MA, et al. Long-term changes in energy expenditure and body composition after massive weight loss induced by gastric bypass surgery. Am J Clin Nutr. 2003;78:22-30.

20. Sjölund K, Sandén G, Håkanson R, et al. Endocrine cells in human intestine: an immunocytochemical study. Gastroenterology. 1983;85:1120-1130

21. Egerod KL, Engelstoft MS, Grunddal KV, et al. A major lineage of enteroendocrine cells coexpress CCK, secretin, GIP, GLP-1, PYY, and neurotensin but not somatostatin. Endocrinology. 2012;153:5782-5795.

22. Grunddal KV, Ratner CF, Svendsen B, et al. Neurotensin is coexpressed, coreleased, and acts together with GLP-1 and PYY in enteroendocrine control of metabolism. Endocrinology. 2016;157:176-194.

23. Sykaras AG, Demenis C, Cheng L, et al. Duodenal CCK cells from male mice express multiple hormones including ghrelin. Endocrinology. 2014;155:3339-3351.

24. Little TJ, Doran S, Meyer JH, et al. The release of GLP-1 and ghrelin, but not GIP and CCK, by glucose is dependent upon the length of small intestine exposed. Am J Physiol Endocrinol Metab. 2006;291:E647-E 655.

25. Psichas A, Reimann F, Gribble FM, et al. Gut chemosensing mechanisms. J Clin Invest. 2015;125:908-917.

26. Moss CE, Glass LL, Diakogiannaki E, et al. Lipid derivatives activate GPR119 and trigger GLP-1 secretion in primary murine L-cells. Peptides. 2016;77:16-20.

27. Reimann F, Tolhurst G, Gribble FM. G-protein-coupled receptors in intestinal chemosensation. Cell Metab. 2012;15:421-431.

28. Wren AM, Small CJ, Abbott CR, et al. Ghrelin causes hyperphagia and obesity in rats. Diabetes. 2001;50:2540-2547.

29. Casajoana A, Pujol J, Garcia A, et al. Predictive value of gut peptides in T2D remission: randomized controlled trial comparing metabolic gastric bypass, sleeve gastrectomy and greater curvature plication. Obes Surg. 2017;27:2235-2245.

30. Nosso G, Griffo E, Cotugno M, et al. Comparative effects of Rouxen- $Y$ gastric bypass and sleeve gastrectomy on glucose homeostasis and incretin hormones in obese type 2 diabetic patients: a one-year prospective study. Horm Metab Res. 2016;48:312-317.

31. Stefater MA, Sandoval DA, Chambers AP, et al. Sleeve gastrectomy in rats improves postprandial lipid clearance by reducing intestinal triglyceride secretion. Gastroenterology. 2011;141:939-949 e4.

32. Peterli R, Steinert RE, Woelnerhanssen B, et al. Metabolic and hormonal changes after laparoscopic Roux-en-Y gastric bypass and sleeve gastrectomy: a randomized, prospective trial. Obes Surg. 2012;22:740-748
33. Lee $\mathrm{W}-\mathrm{J}$, Chen $\mathrm{C}-\mathrm{Y}$, Chong $\mathrm{K}$, et al. Changes in postprandial gut hormones after metabolic surgery: a comparison of gastric bypass and sleeve gastrectomy. Surg Obes Relat Dis. 2011;7:683-690.

34. Hajnal A, Kovacs P, Ahmed T, et al. Gastric bypass surgery alters behavioral and neural taste functions for sweet taste in obese rats. Am J Physiol Gastrointest Liver Physiol. 2010;299:G967-G979.

35. Korner J, Inabnet W, Conwell IM, et al. Differential effects of gastric bypass and banding on circulating gut hormone and leptin levels. Obesity (Silver Spring). 2006;14:1553-1561.

36. Laferrere B, Swerdlow N, Bawa B, et al. Rise of oxyntomodulin in response to oral glucose after gastric bypass surgery in patients with type 2 diabetes. J Clin Endocrinol Metab. 2010;95:4072-4076.

37. Yousseif A, Emmanuel J, Karra E, et al. Differential effects of laparoscopic sleeve gastrectomy and laparoscopic gastric bypass on appetite, circulating acyl-ghrelin, peptide YY3-36 and Active GLP-1 levels in non-diabetic humans. Obes Surg. 2014;24:241-252.

38. Shin AC, Zheng H, Townsend RL, et al. Meal-induced hormone responses in a rat model of Roux-en-Y gastric bypass surgery. Endocrinology. 2010;151:1588-1597.

39. Nannipieri M, Baldi S, Mari A, et al. Roux-en-Y gastric bypass and sleeve gastrectomy: mechanisms of diabetes remission and role of gut hormones. J Clin Endocrinol Metab. 2013;98:4391-4399.

40. Chandarana K, Gelegen C, Karra E, et al. Diet and gastrointestinal bypass-induced weight loss: the roles of ghrelin and peptide $Y Y$. Diabetes. 2011;60:810-818.

41. Chambers AP, Jessen L, Ryan KK, et al. Weight-independent changes in blood glucose homeostasis after gastric bypass or vertical sleeve gastrectomy in rats. Gastroenterology. 2011;141:950-958.

42. Falken $\mathrm{Y}$, Hellstrom PM, Holst JJ, et al. Changes in glucose homeostasis after Roux-en- $Y$ gastric bypass surgery for obesity at day three, two months, and one year after surgery: role of gut peptides. J Clin Endocrinol Metab. 2011;96:2227-2235.

43. Laferrere B, Teixeira J, McGinty J, et al. Effect of weight loss by gastric bypass surgery versus hypocaloric diet on glucose and incretin levels in patients with type 2 diabetes. J Clin Endocrinol Metab. 2008;93:2479-2485.

44. Jorgensen NB, Jacobsen SH, Dirksen C, et al. Acute and long-term effects of Roux-en-Y gastric bypass on glucose metabolism in subjects with Type 2 diabetes and normal glucose tolerance. AJP Endocrinol Metab. 2012;303:E122-E131.

45. Romero F, Nicolau J, Flores L, et al. Comparable early changes in gastrointestinal hormones after sleeve gastrectomy and RouxEn-Y gastric bypass surgery for morbidly obese type 2 diabetic subjects. Surg Endosc. 2012;26:2231-2239.

46. le Roux CW, Borg C, Wallis K, et al. Gut hypertrophy after gastric bypass is associated with increased glucagon-like peptide 2 and intestinal crypt cell proliferation. Ann Surg. 2010;252:50-56.

47. Patel A, Yusta B, Matthews D, et al. GLP-2 receptor signaling controls circulating bile acid levels but not glucose homeostasis in Gcgr-/- mice and is dispensable for the metabolic benefits ensuing after vertical sleeve gastrectomy. Mol Metab. 2018;16:45-54

48. Cheeseman $\mathrm{Cl}$. Upregulation of SGLT-1 transport activity in rat jejunum induced by GLP-2 infusion in vivo. Am J Physiol. 1997;273:R1965-R1971

49. Svane MS, Jørgensen NB, Bojsen-Møller KN, et al. Peptide YY and glucagon-like peptide-1 contribute to decreased food intake after Roux-en-Y gastric bypass surgery. Int J Obes. 2016;40:1699-1706.

50. Tharakan G, Behary P, Wewer Albrechtsen NJ, et al. Roles of increased glycaemic variability, GLP-1 and glucagon in hypoglycaemia after Roux-en-Y gastric bypass. Eur J Endocrinol. 2017;177:455-464

51. Wewer Albrechtsen NJ, Hartmann B, Veedfald S, et al. Hyperglucagonaemia analysed by glucagon sandwich ELISA: 
nonspecific interference or truly elevated levels? Diabetologia. 2014;57:1919-1926.

52. Salehi M, Gastaldelli A, D'Alessio DA. Altered islet function and insulin clearance cause hyperinsulinemia in gastric bypass patients with symptoms of postprandial hypoglycemia. J Clin Endocrinol Metab. 2014;99:2008-2017.

53. Goldfine AB, Mun EC, Devine E, et al. Patients with neuroglycopenia after gastric bypass surgery have exaggerated incretin and in sulin secretory responses to a mixed meal. J Clin Endocrinol Metab. 2007;92:4678-4685.

54. Roberts GP, Kay RG, Howard J, et al. Gastrectomy with Roux-en-Y reconstruction as a lean model of bariatric surgery. Surg Obes Relat Dis. 2018;14:562-568.

55. Mclntyre N, Holsworth DC, Turner DS. New interpretation of oral glucose tolerance. Lancet. 1964;2:20-21.

56. Nauck MA, Bartels E, Orskov C, et al. Additive insulinotropic effects of exogenous synthetic human gastric inhibitory polypeptide and glucagon-like peptide-1-(7-36) amide infused at near-physiological insulinotropic hormone and glucose concentrations. J Clin Endocrinol Metab. 1993;76:912-917.

57. Vilsbøll T, Krarup T, Madsbad S, et al. Both GLP-1 and GIP are insulinotropic at basal and postprandial glucose levels and contribute nearly equally to the incretin effect of a meal in healthy subjects. Regul Pept. 2003;114:115-121.

58. Nauck M, Stöckmann F, Ebert $R$, et al. Reduced incretin effect in type 2 (non-insulin-dependent) diabetes. Diabetologia. 1986;29:46-52

59. Nauck MA, Kind J, Köthe LD, et al. Quantification of the contribution of GLP-1 to mediating insulinotropic effects of DPP-4 inhibition with vildagliptin in healthy subjects and patients with type 2 diabetes using exendin [9-39] as a GLP-1 receptor antagonist. Diabetes. 2016;65:2440-2447.

60. Hansotia T, Baggio LL, Delmeire D, et al. Double incretin receptor knockout (DIRKO) mice reveal an essential role for the enteroinsular axis in transducing the glucoregulatory actions of DPP-IV inhibitors. Diabetes. 2004;53:1326-1335.

61. Svane MS, Bojsen-Møller KN, Nielsen S, et al. Effects of endogenous GLP-1 and GIP on glucose tolerance after Rouxen-Y gastric bypass surgery. Am J Physiol Endocrinol Metab. 2016;310:E505-E514.

62. Jacobsen SH, Olesen SC, Dirksen C, et al. Changes in gastrointestinal hormone responses, insulin sensitivity, and beta-cell function within 2 weeks after gastric bypass in non-diabetic subjects. Obes Surg. 2012;22:1084-1096.

63. Korner J, Bessler M, Inabnet W, et al. Exaggerated glucagon-like peptide-1 and blunted glucose-dependent insulinotropic peptide secretion are associated with Roux-en-Y gastric bypass but not adjustable gastric banding. Surg Obes Relat Dis. 2007;3:597-601.

64. Aung L, Lee W-J, Chen SC, et al. Bariatric surgery for patients with early-onset vs late-onset type 2 diabetes. JAMA Surg. 2016:151:798-805.

65. Jiménez A, Mari A, Casamitjana R, et al. GLP-1 and glucose tolerance after sleeve gastrectomy in morbidly obese subjects with type 2 diabetes. Diabetes. 2014;63:3372-3377.

66. Jørgensen NB, Dirksen C, Bojsen-Møller KN, et al. Exaggerated glucagon-like peptide 1 response is important for improved $\beta$-cell function and glucose tolerance after Roux-en- $Y$ gastric bypass in patients with type 2 diabetes. Diabetes. 2013;62:3044-3052.

67. Salehi M, Prigeon RL, D'Alessio DA. Gastric bypass surgery enhances glucagon-like peptide 1-stimulated postprandial insulin secretion in humans. Diabetes. 2011;60:2308-2314.

68. Jiménez $A$, Ceriello $A$, Casamitjana $R$, et al. Remission of type 2 diabetes after Roux-en-Y gastric bypass or sleeve gastrectomy is associated with a distinct glycemic profile. Ann Surg. $2015 \cdot 261 \cdot 316-322$
69. Mokadem M, Zechner JF, Margolskee RF, et al. Effects of Rouxen-Y gastric bypass on energy and glucose homeostasis are preserved in two mouse models of functional glucagon-like peptide-1 deficiency. Mol Metab. 2014;3:191-201.

70. Wilson-Pérez HE, Chambers AP, Ryan KK, et al. Vertical sleeve gastrectomy is effective in two genetic mouse models of glucagonlike peptide-1 receptor deficiency. Diabetes. 2013;62:2380-2385.

71. Ye J, Hao Z, Mumphrey MB, et al. GLP-1 receptor signaling is not required for reduced body weight after RYGB in rodents. Am J Physiol Regul Integr Comp Physiol. 2014;306:R352-R362.

72. Garibay D, McGavigan AK, Lee SA, et al. $\beta$-cell glucagon-like peptide-1 receptor contributes to improved glucose tolerance after vertical sleeve gastrectomy. Endocrinology. 2016;157:3405-3409.

73. Douros JD, Lewis AG, Smith EP, et al. Enhanced glucose control following vertical sleeve gastrectomy does not require a $\beta$-Cell glucagon-like peptide 1 receptor. Diabetes. 2018;67:1504-1511.

74. Larraufie P, Roberts GP, McGavigan AK, et al. Important role of the GLP-1 axis for glucose homeostasis after bariatric surgery. Cell Rep. 2019;26:1399-1408.e6.

75. DeVries JH. Glucose variability: where it is important and how to measure it. Diabetes. 2013;62:1405-1408.

76. Jiménez A, Casamitjana R, Viaplana-Masclans J, et al. GLP-1 action and glucose tolerance in subjects with remission of type 2 diabetes after gastric bypass surgery. Diabetes Care. 2013;36:2062-2069.

77. Bobbioni-Harsch E, Huber O, Morel P, et al. Factors influencing energy intake and body weight loss after gastric bypass. Eur J Clin Nutr. 2002;56:551-556.

78. Dias MCG, Ribeiro AG, Scabim VM, et al. Dietary intake of female bariatric patients after anti-obesity gastroplasty. Clinics (Sao Paulo). 2006;61:93-98.

79. Wardé-Kamar J, Rogers M, Flancbaum L, et al. Calorie intake and meal patterns up to 4 years after Roux-en-Y gastric bypass surgery. Obes Surg. 2004;14:1070-1079.

80. Moize V, Geliebter A, Gluck ME, et al. Obese patients have inadequate protein intake related to protein intolerance up to 1 year following Roux-en-Y gastric bypass. Obes Surg. 2003;13:23-28.

81. Trostler N, Mann A, Zilberbush N, et al. Nutrient intake following vertical banded gastroplasty or gastric bypass. Obes Surg. 1995;5:403-410.

82. Näslund I, Järnmark I, Andersson H. Dietary intake before and after gastric bypass and gastroplasty for morbid obesity in women. Int J Obes. 1988;12:503-513.

83. Brolin RE, Robertson LB, Kenler HA, et al. Weight loss and dietary intake after vertical banded gastroplasty and Roux-en-Y gastric bypass. Ann Surg. 1994;220:782-790.

84. Shin AC, Zheng H, Pistell PJ, et al. Roux-en-Y gastric bypass surgery changes food reward in rats. Int J Obes. 2011;35(5): 642-651.

85. Zheng $H$, Shin AC, Lenard NR, et al. Meal patterns, satiety, and food choice in a rat model of Roux-en-Y gastric bypass surgery. Am J Physiol Regul Integr Comp Physiol. 2009;297:R1273-R1282.

86. Mathes CM, Bohnenkamp RA, Blonde GD, et al. Gastric bypass in rats does not decrease appetitive behavior towards sweet or fatty fluids despite blunting preferential intake of sugar and fat. Physiol Behav. 2015;142:179-188.

87. Ullrich J, Ernst B, Wilms B, et al. Roux-en $Y$ gastric bypass surgery reduces hedonic hunger and improves dietary habits in severely obese subjects. Obes Surg. 2013;23:50-55.

88. Makaronidis JM, Neilson S, Cheung W-H, et al. Reported appetite, taste and smell changes following Roux-en-Y gastric bypass and sleeve gastrectomy: effect of gender, type 2 diabetes and relationship to post-operative weight loss. Appetite. 2016;107:93-105.

89. Zerrweck C, Zurita L, Álvarez G, et al. Taste and olfactory changes following laparoscopic gastric bypass and sleeve gastrectomy. Obes Surg. 2016;26:1296-1302. 
90. le Roux CW, Bueter M, Theis N, et al. Gastric bypass reduces fat intake and preference. AJP Regul Integr Comp Physiol. 2011;301:R1057-R1066.

91. Boxwell AJ, Chen Z, Mathes CM, et al. Effects of high-fat diet and gastric bypass on neurons in the caudal solitary nucleus. Physiol Behav. 2015;152:329-339.

92. Hunt KF, Dunn JT, le Roux CW, et al. Differences in regional brain responses to food ingestion after Roux-en-Y gastric bypass and the role of gut peptides: a neuroimaging study. Diabetes Care. 2016;39:1787-1795.

93. Halperin F, Patti ME, Skow M, et al. Continuous glucose monitoring for evaluation of glycemic excursions after gastric bypass. J Obes. 2011;2011:869536.

94. Shin AC, Zheng H, Berthoud H-R. Vagal innervation of the hepatic portal vein and liver is not necessary for Roux-en-Y gastric bypass surgery-induced hypophagia, weight loss, and hypermetabolism. Ann Surg. 2012;255:294-301.

95. Hankir MK, Seyfried F, Hintschich CA, et al. Gastric bypass surgery recruits a gut PPAR- $\alpha$-striatal D1R pathway to reduce fat appetite in obese rats. Cell Metab. 2017;25:335-344.

96. Bueter $\mathrm{M}$, Löwenstein $\mathrm{C}$, Ashrafian $\mathrm{H}$, et al. Vagal sparing surgical technique but not stoma size affects body weight loss in rodent model of gastric bypass. Obes Surg. 2010;20:616-622.

97. Hao Z, Townsend RL, Mumphrey MB, et al. Vagal innervation of intestine contributes to weight loss after Roux-en- $Y$ gastric bypass surgery in rats. Obes Surg. 2014;24:2145-2151.

98. Mansuy-Aubert V, Gautron L, Lee S, et al. Loss of the liver X receptor $L X R \alpha / \beta$ in peripheral sensory neurons modifies energy expenditure. Elife. 2015;4:doi.org/10.7554/elife.06667.

99. Liu C, Bookout AL, Lee $\mathrm{S}$, et al. PPAR in vagal neurons regulates high-fat diet induced thermogenesis. Cell Metab. 2014;19:722-730.

100. Williams EK, Chang RB, Strochlic DE, et al. Sensory neurons that detect stretch and nutrients in the digestive system. Cell. 2016;166:209-221.

101. Dailey MJ. Nutrient-induced intestinal adaption and its effect in obesity. Physiol Behav. 2014;136:74-78.

102. Mumphrey MB, Hao Z, Townsend RL, et al. Sleeve gastrectomy does not cause hypertrophy and reprogramming of intestinal glucose metabolism in rats. Obes Surg. 2015;25:1468-1473.

103. Mumphrey MB, Patterson LM, Zheng $H$, et al. Roux-en- $Y$ gastric bypass surgery increases number but not density of CCK-, GLP-1-, 5-HT-, and neurotensin-expressing enteroendocrine cells in rats. Neurogastroenterol Motil. 2013;25:e70-e79.

104. Hansen CF, Bueter M, Theis N, et al. Hypertrophy dependent doubling of L-cells in Roux-en-Y gastric bypass operated rats. PLoS ONE. 2013;8:e65696.

105. Nguyen NQ, Debreceni TL, Bambrick JE, et al. Rapid gastric and intestinal transit is a major determinant of changes in blood glucose, intestinal hormones, glucose absorption, and postprandial symptoms after gastric bypass. Obesity (Silver Spring). 2014;22:2003-2009.

106. Cavin J-B, Couvelard A, Lebtahi R, et al. Differences in alimentary glucose absorption and intestinal disposal of blood glucose following Roux-en-Y Gastric bypass vs sleeve gastrectomy. Gastroenterology. 2016;50:454-464.e9.

107. Myronovych A, Salazar-Gonzalez R-M, Ryan KK, et al. The role of small heterodimer partner in nonalcoholic fatty liver disease improvement after sleeve gastrectomy in mice. Obesity. 2014;22:2301-2311.

108. Nausheen S, Shah IH, Pezeshki A, et al. Effects of sleeve gastrectomy and ileal transposition, alone and in combination, on food intake, body weight, gut hormones, and glucose metabolism in rats. AJP Endocrinol Metab. 2013;305:E507-E518.

109. Haeusler RA, Astiarraga B, Camastra $S$, et al. Human insulin resistance is associated with increased plasma levels of $12 \alpha$-hydroxylated bile acids. Diabetes. 2013;62:4184-4191.
110. Wewalka M, Patti M-E, Barbato $C$, et al. Fasting serum taurineconjugated bile acids are elevated in type 2 diabetes and do not change with intensification of insulin. J Clin Endocrinol Metab. 2014;99:1442-1451.

111. Sonne DP, van Nierop FS, Kulik W, et al. Postprandial plasma concentrations of individual bile acids and FGF-19 in patients with type 2 diabetes. J Clin Endocrinol Metab. 2016;101:3002-3009.

112. Patti M-E, Houten SM, Bianco AC, et al. Serum bile acids are higher in humans with prior gastric bypass: potential contribution to improved glucose and lipid metabolism. Obesity (Silver Spring). 2009;17:1671-1677.

113. Kohli R, Bradley D, Setchell KD, et al. Weight loss induced by roux-en-y gastric bypass but not laparoscopic adjustable gastric banding increases circulating bile acids. J Clin Endocrinol Metab. 2013;98:E708-E712.

114. Pournaras DJ, Glicksman C, Vincent RP, et al. The role of bile after Roux-en-Y gastric bypass in promoting weight loss and improving glycaemic control. Endocrinology. 2012;153:3613-3619.

115. Sachdev S, Wang Q, Billington C, et al. FGF 19 and bile acids increase following Roux-en- $Y$ gastric bypass but not after medical management in patients with type 2 diabetes. Obes Surg. 2016;26:957-965.

116. Steinert RE, Peterli R, Keller S, et al. Bile acids and gut peptide secretion after bariatric surgery: a 1-year prospective randomized pilot trial. Obesity (Silver Spring). 2013;21:E660-E668.

117. $\mathrm{Yu} \mathrm{H}, \mathrm{Ni} \mathrm{Y}, \mathrm{Bao} \mathrm{Y}$, et al. Chenodeoxycholic acid as a potential prognostic marker for Roux-en- $Y$ gastric bypass in chinese obese patients. J Clin Endocrinol Metab. 2015;100:4222-4230.

118. Ryan KK, Tremaroli V, Clemmensen C, et al. FXR is a molecular target for the effects of vertical sleeve gastrectomy. Nature. 2014;509:183-188.

119. Myronovych A, Kirby M, Ryan KK, et al. Vertical sleeve gastrectomy reduces hepatic steatosis while increasing serum bile acids in a weight-loss-independent manner. Obesity (Silver Spring). 2014;22:390-400.

120. Ozcan U, Yilmaz E, Ozcan L, et al. Chemical chaperones reduce ER stress and restore glucose homeostasis in a mouse model of type 2 diabetes. Science. 2006;313:1137-1140.

121. Nielsen S, Svane MS, Kuhre RE, et al. Chenodeoxycholic acid stimulates glucagon-like peptide-1 secretion in patients after Rouxen-Y gastric bypass. Physiol Rep. 2017;5:e13140.

122. Kawamata Y, Fujii R, Hosoya M, et al. A G protein-coupled receptor responsive to bile acids. J Biol Chem. 2003;278:9435-9440.

123. Parks DJ, Blanchard SG, Bledsoe RK, et al. Bile acids: natural ligands for an orphan nuclear receptor. Science. 1999;284:1365-1368.

124. Makishima M, Okamoto AY, Repa JJ, et al. Identification of a nuclear receptor for bile acids. Science. 1999;284:1362-1365.

125. Katsuma S, Hirasawa A, Tsujimoto G. Bile acids promote glucagon-like peptide-1 secretion through TGR5 in a murine enteroendocrine cell line STC-1. Biochem Biophys Res Commun. 2005;329: 386-390.

126. Watanabe M, Houten SM, Mataki C, et al. Bile acids induce energy expenditure by promoting intracellular thyroid hormone activation. Nature. 2006;439:484-489.

127. Cariou B, van Harmelen K, Duran-Sandoval D, et al. The farnesoid $X$ receptor modulates adiposity and peripheral insulin sensitivity in mice. J Biol Chem. 2006;281:11039-11049.

128. Cipriani S, Mencarelli A, Palladino G, et al. FXR activation reverses insulin resistance and lipid abnormalities and protects against liver steatosis in Zucker (fa/fa) obese rats. J Lipid Res. 2010;51:771-784.

129. Ma K, Saha PK, Chan L, et al. Farnesoid X receptor is essential for normal glucose homeostasis. J Clin Invest. 2006;116:1102-1109.

130. Morton GJ, Matsen ME, Bracy DP, et al. FGF19 action in the brain induces insulin-independent glucose lowering. J Clin Invest. 2013;123:4799-4808. 
131. Fu L, John LM, Adams SH, et al. Fibroblast growth factor 19 increases metabolic rate and reverses dietary and leptin-deficient diabetes. Endocrinology. 2004;145:2594-2603.

132. Kir S, Beddow SA, Samuel VT, et al. FGF19 as a postprandial, insulin-independent activator of hepatic protein and glycogen synthesis. Science. 2011;331:1621-1624.

133. Nies VJM, Sancar G, Liu W, et al. Fibroblast growth factor signaling in metabolic regulation. Front Endocrinol (Lausanne). 2016;6:193.

134. Gerhard GS, Styer AM, Wood GC, et al. A role for fibroblast growth factor 19 and bile acids in diabetes remission after Roux-en-Y gastric bypass. Diabetes Care. 2013;36:1859-1864.

135. McGavigan AK, Garibay D, Henseler ZM, et al. TGR5 contributes to glucoregulatory improvements after vertical sleeve gastrectomy in mice. Gut. 2017;66:226-234.

136. Ding L, Sousa KM, Jin L, et al. Vertical sleeve gastrectomy activates GPBAR-1/TGR5 to sustain weight loss, improve fatty liver, and remit insulin resistance in mice. Hepatology. 2016;64:760-773.

137. Vassileva G, Golovko A, Markowitz L, et al. Targeted deletion of Gpbar1 protects mice from cholesterol gallstone formation. Biochem J. 2006;398:423-430.
138. Parséus A, Sommer N, Sommer F, et al. Microbiota-induced obesity requires farnesoid X receptor. Gut. 2017;66:429-437.

139. Sayin SI, Wahlström A, Felin J, et al. Gut microbiota regulates bile acid metabolism by reducing the levels of tauro-beta-muricholic acid, a naturally occurring FXR antagonist. Cell Metab. 2013;17:225-235.

140. Aron-Wisnewsky J, Prifti E, Belda E, et al. Major microbiota dysbiosis in severe obesity: fate after bariatric surgery. Gut. 2019;68:70-82.

How to cite this article: Sandoval DA. Mechanisms for the metabolic success of bariatric surgery. J Neuroendocrinol. 2019;31:e12708. https://doi.org/10.1111/jne.12708 